\title{
Male Reproductive System Precancerous Condition
}

National Cancer Institute

\section{Source}

National Cancer Institute. Male Reproductive System Precancerous Condition. NCI

Thesaurus. Code C38164.

A premalignant pathologic process arising from an organ of the male reproductive system. It is characterized by cellular atypia and/or dysplasia but does not show morphologic evidence of stromal invasion at the time of diagnosis. Representative examples include prostatic intraepithelial neoplasia, giant condyloma acuminatum of the penis, and penile intraepithelial neoplasia. 